NOVA

University of Newcastle Research Online

nova.newcastle.edu.au

Kouretzis, George P.; Sheng, Daichao; Sloan, Scott W. "Sand-pipeline-trench lateral interaction effects for shallow buried pipelines" Computers and Geotechnics Vol. 54, p. 53-59 (2013)

Available from: http://dx.doi.org/10.1016/j.compgeo.2013.05.008

Accessed from: http://hdl.handle.net/1959.13/1056706 


\title{
Sand-pipeline-trench lateral interaction effects for shallow buried pipelines
}

\author{
George P. Kouretzis ${ }^{1}$, Daichao Sheng and Scott W. Sloan \\ ARC Centre of Excellence for Geotechnical Science and Engineering, Faculty of Engineering \\ and Built Environment, The University of Newcastle, Callaghan, NSW 2308, Australia
}

A large-deformation numerical methodology is applied to simulate the interaction effects for a pipeline installed in a trench backfilled with loosely deposited dry sand, focusing on shallow buried pipelines subjected to lateral displacements relative to the surrounding soil. Based on the backfill-pipeline deformation mode under shallow embedment conditions, described in previous experimental studies, analyses are performed while considering only the critical state shear strength parameters of the backfill. The numerical methodology is validated against experimental full-scale test measurements from the literature, for pipelines buried in uniform dry loose and medium sand. Parametric analyses are performed to generate approximate formulas and charts for calculating i) the maximum force on the pipeline, and ii) the minimum trench dimensions to eliminate interaction with the surrounding natural ground. Application of the proposed approach in the prediction of independent full-scale test results for a pipeline embedded in a shallow trench demonstrates its effectiveness, and underlines the effect of trench dimensioning on the response of the pipeline.

Keywords: buried pipelines, trench, dry sand, embedment, lateral movement, design

\footnotetext{
${ }^{1}$ Corresponding author - email: Georgios.Kouretzis@newcastle.edu.au tel. +61 24921 6449, postal address: EA Building, University of Newcastle, Callaghan NSW 2308, Australia
} 


\section{Introduction}

Various experimental and numerical studies have been published on the quantification of soil-buried pipeline interaction effects due to the static movement of a pipeline relative to its surrounding soil. Such movements may result from seismic fault rupture, slope instability, ground subsidence due to underground works, or liquefaction [1], and will result in additional forces being applied on the pipeline. We focus our attention on horizontal relative movement, since this is generally most critical case for the integrity of a pipeline, as it tends to result in higher forces compared to axial or upward movement. Common practice for the calculation of the peak horizontal force per unit length is based on the following expression:

$$
F=\gamma H N_{h} D
$$

where $Y^{\prime}$ is the effective unit weight of the soil, $H$ is the depth to the centerline of the pipe, $D$ is the external pipeline diameter, and $N_{h}$ is a dimensionless parameter depending on the soil friction angle, $\varphi$, and the embedment ratio, $H / D$. Calculation of the dimensionless parameter $N_{h}$ is commonly based either on the nomograph proposed by Hanshen [2, 3], or on a similar nomograph proposed in the benchmark experimental study by Trautmann and O' Rourke [1]. The latter was compiled from the results of a series of tests on pipelines in dry sand, for embedment ratios ranging from $H / D=1.5$ to $H / D=11$. More recent studies by Olson [4], O'Rourke [5], Yimsiri et al. [6], di Prisco and Galli [7], Turner [8], and Paulin et al. [9] deal with additional factors such as deep embedment conditions, the effect of sand water content, and the response under cyclic displacements.

Common construction practice for placed in-situ buried pipelines includes installing the pipeline in a relatively shallow trench, which is subsequently backfilled with sand. The backfill material is loosely deposited since, in case of relative movement, higher backfill densities will result in higher forces applied to the pipeline (Eq. 1). A rather 
conservative design approach of assuming medium-density sand for the calculation of the pipeline forces could be justified as, during the lifetime of the pipeline, the backfill could be unintentionally compacted due to traffic loads, nearby machine vibrations, seismic wave propagation etc. The dimensions of the trench must be "adequate", so that the pipeline response will depend solely on the properties of the controlled backfill material, and not on the perhaps much stiffer surrounding soil, as stated in ASCE-ALA "Guidelines for the design of buried steel pipe" [3]. To the author's knowledge, however, there is no robust method for quantitatively predicting appropriate trench dimensions to meet this requirement. The relevant experimental studies [10] examine limited trench configurations, and do not propose design guidelines.

Apart from the above, existing studies on the force-displacement response of pipelines $[1,5,6]$ treat a very wide range of embedment depths and backfill properties under a common assumption framework. It should be noted, however, that the kinematic mechanism under large relative displacements changes from a global "flow-type" failure under shallow embedment conditions, to a local shear soil failure under deep embedment conditions [6].

This paper focuses on the common case of buried pipelines embedded in shallow trenches (up to $H / D=5.5$ ), where a "flow-type" failure is expected, for loose-tomedium dry sand backfills (as per construction practice where differential movements are expected). By employing a numerical model to replicate the experiments performed by Trautmann and O'Rourke [1] in uniform sand, new insight is provided on the parameters affecting the forces developed on the pipeline. Accordingly, a series of parametric analyses are performed to complement existing experimental data for additional embedment ratio cases. Interpretation of the results leads to a refined formula for calculating the dimensionless parameter in Eq. 1, specifically for shallow embedment ratios and loose-to-medium sands. Design charts are also 
developed for determining the minimum trench dimensions that will prevent interaction of the pipeline with the surrounding natural soil. The theoretical findings are compared with the independent experimental results given by Karimian et al. [10] for a pipeline moving horizontally in a uniform backfill inside a trench.

\section{Description and verification of the numerical model}

As the quasi-static relative movement of a pipeline can be of the order of the pipeline diameter, the numerical simulation of pipeline-backfill interaction effects must properly account for the development of large soil deformations. The numerical code ABAQUS/Explicit [11] is employed in the analyses for this purpose. ABAQUS/Explicit utilizes explicit integration to treat highly nonlinear problems, together with the Arbitrary-Lagrangian-Eulerian (ALE) remeshing technique to compensate for the inevitable mesh distortion. The problem is simulated dynamically, but the displacement on the pipeline is applied at a very slow rate of about $0.05 \mathrm{~mm} / \mathrm{sec}$ to avoid numerical instabilities. The simple Mohr-Coulomb constitutive model is used to model the backfill behavior for reasons explained later.

In the first stage of this study, eight (8) basic analyses are performed to replicate the pull tests performed by Trautmann and O'Rourke [1] for a pipeline with an external diameter of $D=0.102 \mathrm{~m}$, buried in uniform loose $\left(\gamma=14.8 \mathrm{kN} / \mathrm{m}^{3}\right)$ and medium $\left(y=16.4 \mathrm{kN} / \mathrm{m}^{3}\right)$ Cornell filter sand. These unit weight values correspond to relative densities of $D_{r}=0 \%$ and $45 \%$, respectively. In the tests simulated, the pipeline is embedded at depths of $H / D=1.5, H / D=3.5, H / D=5.5$, and $H / D=11$.

According to Tratumann and O'Rourke [1], the abovementioned density states correspond to sand peak friction angles of $\varphi_{\text {peak }}=31^{\circ}$ and $36^{\circ}$ for the loose and medium sand, respectively. Thus the critical friction angle of the sand used in the tests is set to $\varphi_{\text {crit }}=31^{\circ}$. Yimsiri et al. [6] estimated the dilatancy angle corresponding to the peak friction angle, $\psi$, from the expression $\varphi_{\text {peak }}=\varphi_{\text {crit }}+0.8 \psi[12]$; which 
suggests that $\psi$ increases from $\psi=0^{\circ}$ to $\psi=5^{\circ}$, as the density of the sand increases from $D_{r}=0 \%$ to $45 \%$.
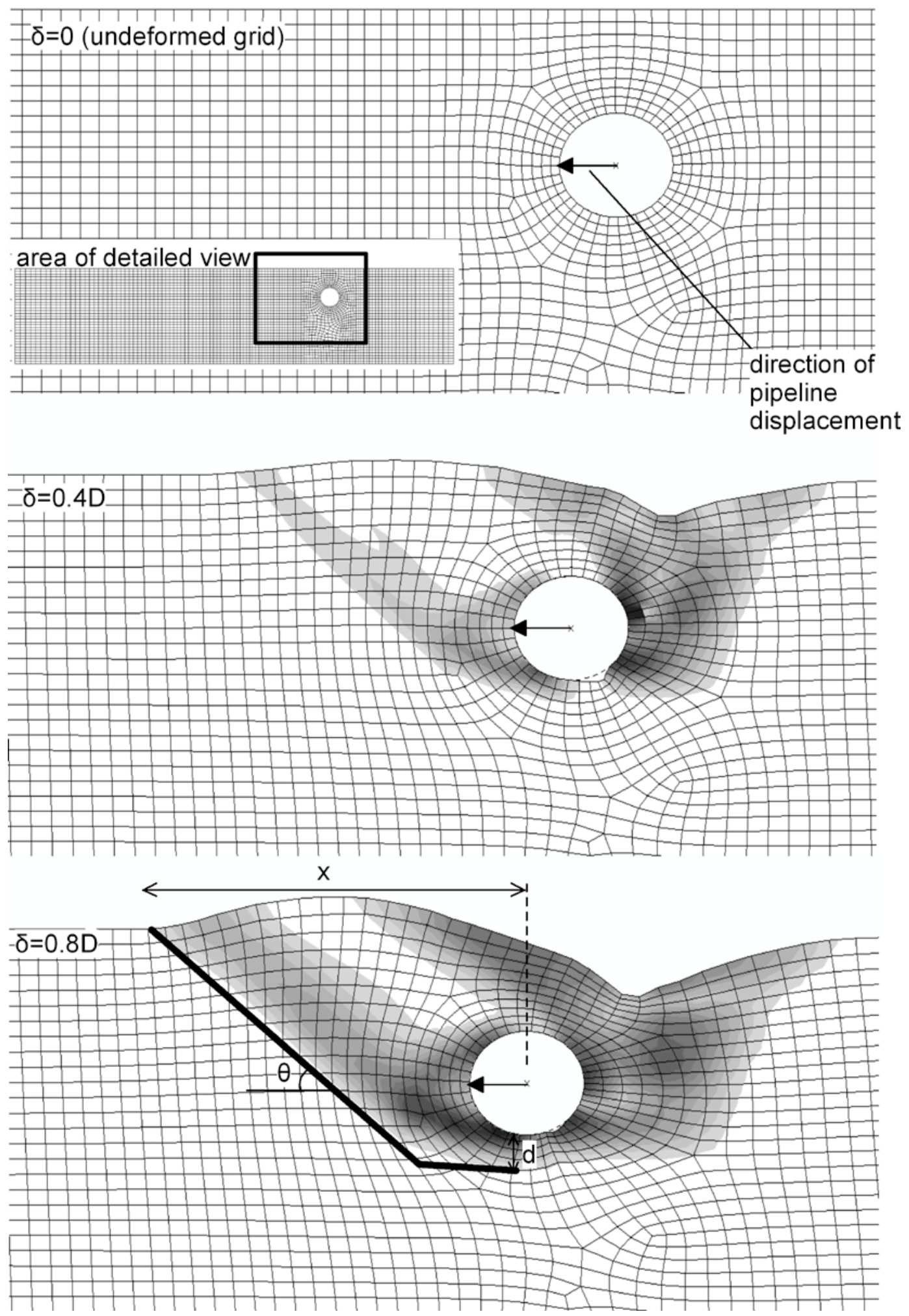

Fig.1 
The abovementioned shear strength parameters were derived from direct shear (DS) tests. To be used in a 2-D plane strain numerical model with the Mohr-Coulomb failure surface, the equivalent plane-strain friction angle, $\varphi_{P S}$, must be estimated since the direct shear test peak stress state does not correspond to a point on the Mohr circle that is tangent to the failure surface [5]. Following Davis [13], the planestrain friction angle can be estimated from the results of direct shear tests, assuming coaxiality of the stress and strain increments, using the expression:

$$
\tan \varphi_{D S}=\frac{\cos \psi \cdot \sin \varphi_{\text {peak }, P S}}{1-\sin \psi \cdot \sin \varphi_{\text {peak }, P S}}
$$

At the critical state, where the dilatancy angle $\psi$ is zero, we have:

$$
\tan \varphi_{\text {crit,DS }}=\sin \varphi_{\text {crit,PS }}
$$

For the numerical simulation of pipe-sand interaction effects, Trautmann and O'Rourke [14] suggest that the use of the peak shear strength parameters from Eq. (2) will provide an upper bound on the forces developing on the pipeline, while the critical state shear strength parameters resulting from Eq. (3) with $\varphi_{\text {crit,PS }}=37^{\circ}$ and $\psi=0^{\circ}$ will provide a lower bound. This is a reasonable assumption if we consider the whole range of embedment depths and sand density values tested by Trautmann and O'Rourke [1], but if we focus on loose-to-medium sands and low H/D ratios providing little confinement, where a large-deformation "flow-type" failure surface is expected to form (Fig. 1), the response of the backfill is expected to primarily depend on its critical strength parameters. This is the rationale behind selecting the MohrCoulomb constitutive model, together with critical state strength parameters, to describe the sand shear behavior, irrespective of its initial density state. Since the critical state friction angle depends chiefly on the sand particle mineralogy and shape [15], and generally ranges between $\varphi_{\text {crit }}=30^{\circ}-33^{\circ}$, the results presented here are not restricted exclusively to Cornell filter sand. 


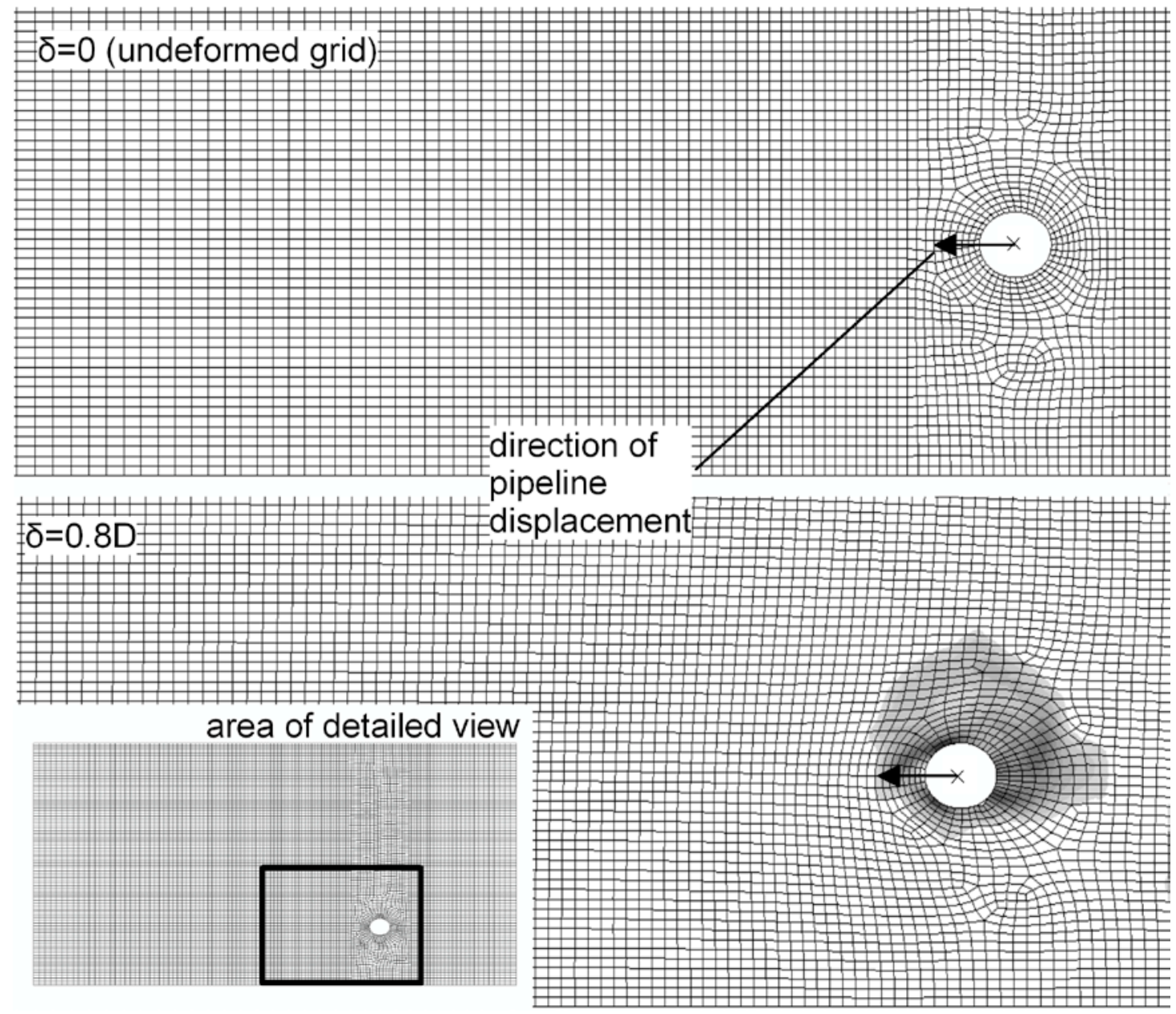

Fig. 2

Some evidence of this fact can be provided via comparisons with results from independent tests, and presented in the following paragraphs.

The backfill Young's modulus at the depth of the pipe centerline, $E$, is estimated as a function of the soil dry unit weight, $\gamma$, and the vertical stress at depth $H, \sigma_{v}$, according to the expression proposed by O' Rourke [5]:

$$
E=2 \cdot 10^{-13.97}\left(\gamma \cdot \sigma_{v}^{0.0378}\right)^{13.7}
$$

where $\gamma$ and $\sigma_{v}$ are expressed in units of $\mathrm{kN} / \mathrm{m}^{3}$ and $\mathrm{kPa}$, respectively. According to O'Rourke [5], the values of $E$ resulting from Eq. (4), which was derived from multiple linear regression analysis of full-scale pipeline tests results, are suitable to be used 
directly in combination with the Mohr-Coulomb model. The Poisson's ratio of the sand is taken as $v=1 / 3$, as in Yimsiri et al. [6] analyses.

The finite element mesh consists of 4,172 4-noded reduced integration elements (CAX4R) with hourglassing control for the $H / D=1.5$ experiment (Fig. 1), and up to 11,757 elements for the $H / D=11$ experiment (Fig. 2). The dimensions of the mesh are equal to the dimensions of the test box, with the pipeline being simulated using rigid elements [6], to which a uniform horizontal displacement is applied. The friction coefficient at the soil-pipeline interface is assumed equal to $\tan \left(0.5 \varphi_{\text {crit,PS }}\right)[6]$. Note that Yimsiri et al. [6] calculated just an 8\% increase in the peak forces applied on the pipeline as the friction coefficient at the interface increases from $\tan \left(0.5 \varphi_{\text {peak }}\right)$ to $\tan \left(1.0 \varphi_{\text {peak }}\right)$. This suggests that the results are not particularly sensitive to the interface friction.

As it is depicted in Fig. 1, a "flow-type" failure occurs in the shallow embedment case. The mobilized soil mass can be identified clearly from the results, allowing the backfill zone which interacts with the pipeline to be determined. A fully-formed active failure wedge is developed upstream for relatively low relative displacement values. On the other hand, as expected, full mobilization of the downstream passive failure wedge develops for larger displacement values. The "flow" of the backfill material has a beneficial effect on the forces generated on the pipeline.

A different failure pattern is observed in the deep embedment case (Fig. 2), where a "local" shear failure develops in the sand around the pipeline, due to the relatively high confinement conditions.

The force-displacement curves from the analyses are compared against the experimental measurements of Trautmann and O'Rourke [1] in Fig. 3. In the same plot, the results of similar numerical analyses performed by Yimsiri et al. [6] are presented (where available), although it should be noted that their study focused on 

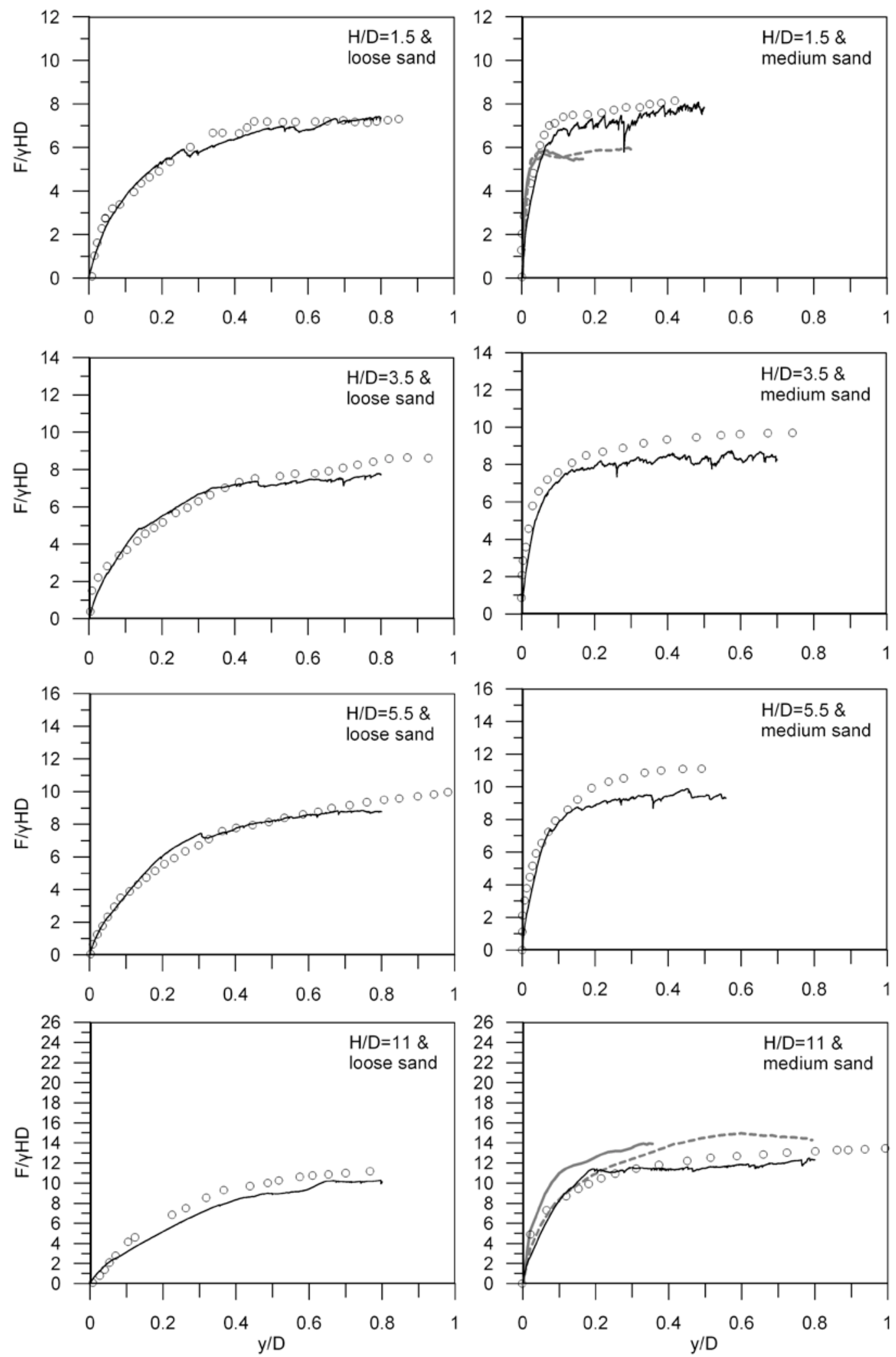

$\circ \circ \circ$ Trautmann \& O' Rourke [1] (measurements)

Yimsiri et al. [6] (Nor-Sand)

Yimsiri et al. [6] (Mohr-Coulomb)

this study

Fig. 3 
deep embedment ratios. The Yimsiri et al. [6] analyses were performed using both the Mohr-Coulomb and Nor-Sand [16] models, with the parameters of the latter being calibrated from the results of experiments in dense sand for $H / D=1.5$ and $H / D=11$.

A fair match is observed between the current numerical predictions and the experimental measurements, implying that the forces on a pipeline embedded in loose-to-medium backfills are not strongly correlated to the peak shear strength characteristics of the sand (i.e. the peak friction angle and dilatancy angle).

\section{Parametric results and practical findings}

The next stage of this study involves twelve (12) additional parametric numerical analyses to fill in the gap between the embedment ratios $H / D=1.5$ and $H / D=5.5$ in the experimental results of Trautmann and O'Rourke [1]. In these analyses, the pipeline is assumed to be embedded in loose and medium sand, with the properties described in the previous paragraph. The purpose of these analyses is twofold:

- To calculate the maximum force applied on the pipeline, and propose a refined method to calculate the dimensionless parameter $N_{h}$ (Eq. 1) for pipelines buried in shallow trenches.

- To define the geometry of the passive failure wedge (Fig. 1) for each of these cases. This will, in turn, define the geometry of the trench in which the pipeline is constructed so as that the force-displacement response remains unaffected by the properties of the natural soil outside the trench. 

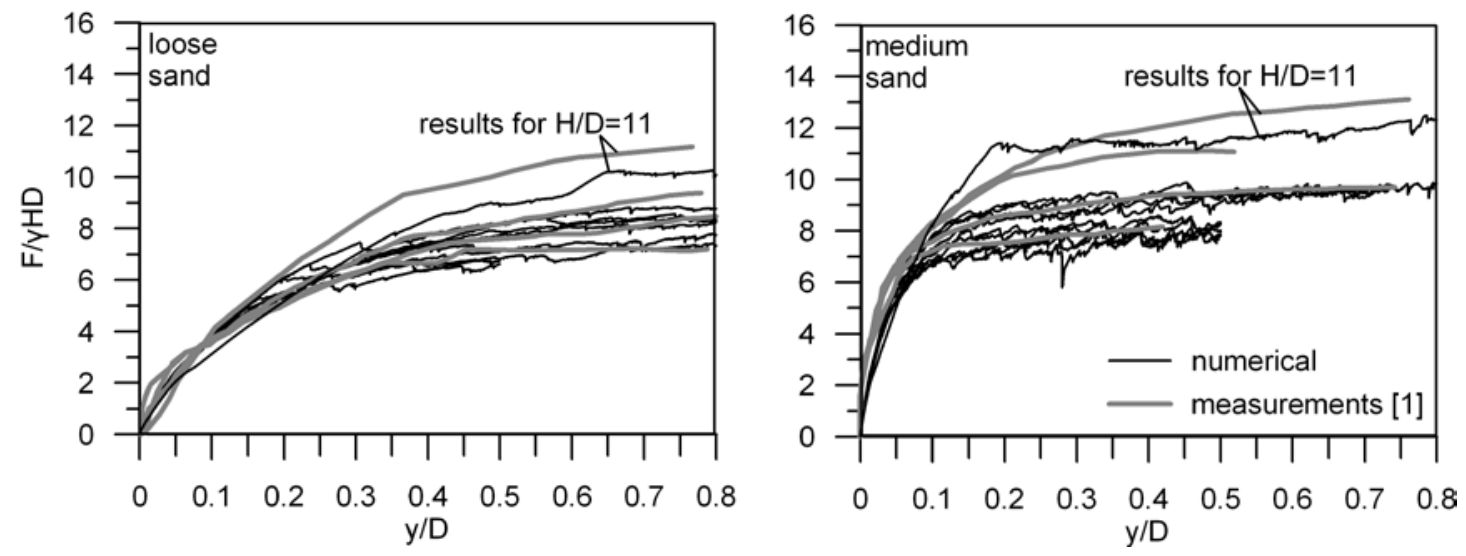

Fig. 4

Fig. 4 shows plots of the dimensionless force $(F / y H D)$ versus normalized displacement $(y / D)$ for all the "shallow" $H / D \leq 5.5$ ratios analyzed, together with the corresponding experimental measurements. Notice that the normalized "yield" displacement, which corresponds to the displacement value where the force on the pipeline reaches a plateau, is more or less insensitive to the value of the $H / D$ ratio, for shallow embedment cases. As expected, this transition is more clearly defined for the medium sand case. Note that the inevitable numerical noise, which is due to the integration scheme and the frequent regeneration of the mesh every about $0.5 \mathrm{~mm}$ of applied displacement, was intentionally not filtered from the results presented in Fig. 4 (and Fig. 3).

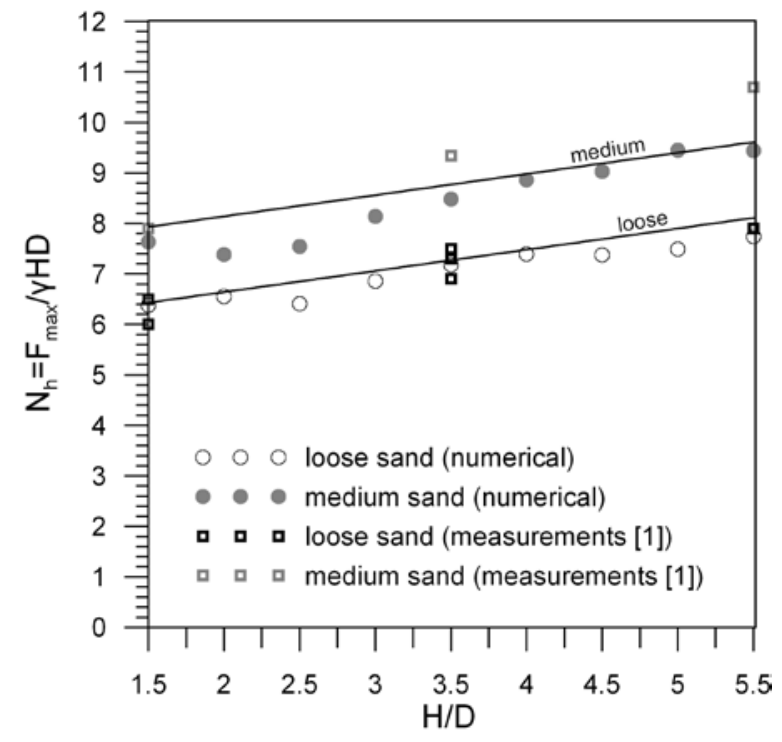

Fig. 5 
Fig. 5 shows a plot of the dimensionless parameter $N_{h}=F_{\max } / \gamma H D$ for the range of embedment ratios considered, together with the relevant experimental measurements by Trautmann and O' Rourke [1]. The analyses corresponding to different sand densities are marked with different symbols. For design purposes, an upper bound estimate of the $N_{h}$ parameter can be assumed for each sand density (Fig. 5). This graph could be considered as a "zoom" of the analogous graphs proposed by Trautmann and O'Rourke [1] and the ASCE-ALA [3] guidelines, which apply to embedment ratios up to $H / D=20$; yet here the backfill material response is quantified via its unit weight only since the results are not strongly dependent on the peak friction angle, $\varphi_{\text {peak }}$. This is an advantage for practical design applications, as it eliminates the need for determining (or estimating) the peak friction angle of the backfill through laboratory testing or empirical correlations.

On the basis of the above, an approximate design formula can be derived using linear interpolation of the maximum force values for different densities. This provides the dimensionless factor $N_{h}$ as a function of a) the embedment ratio, H/D and b) the ratio of the unit weight of the backfill sand over the unit weight of the backfill for $D_{r}=0 \%\left(\gamma_{\text {ref }}\right)$ according to:

$$
N_{h}=0.42\left(\frac{H}{D}\right)+13.8\left(\frac{\gamma}{\gamma_{\text {ref }}}\right)-8.1
$$

The mobilized soil mass geometry, illustrated in Fig. 1, suggests that the dimensions of a trench which encloses the failure prism can be determined by three basic factors: (i) the dimensionless semi-width of the trench, $x / H$, which includes the maximum anticipated horizontal pipeline displacement, $y_{\max }$; (ii) the inclination of the backfill slope, and (iii) the dimensionless distance of the pipeline from the bottom of the trench, $d / D$, which is smaller than one pipeline radius and generally insensitive to the other parameters. Based on the interpretation of contour plots similar to those in 
Fig. 1, these three factors are estimated and presented in Fig. 6. The solid lines drawn in Fig. 6 may be used in practical applications as upper bound values for loose and medium sands.
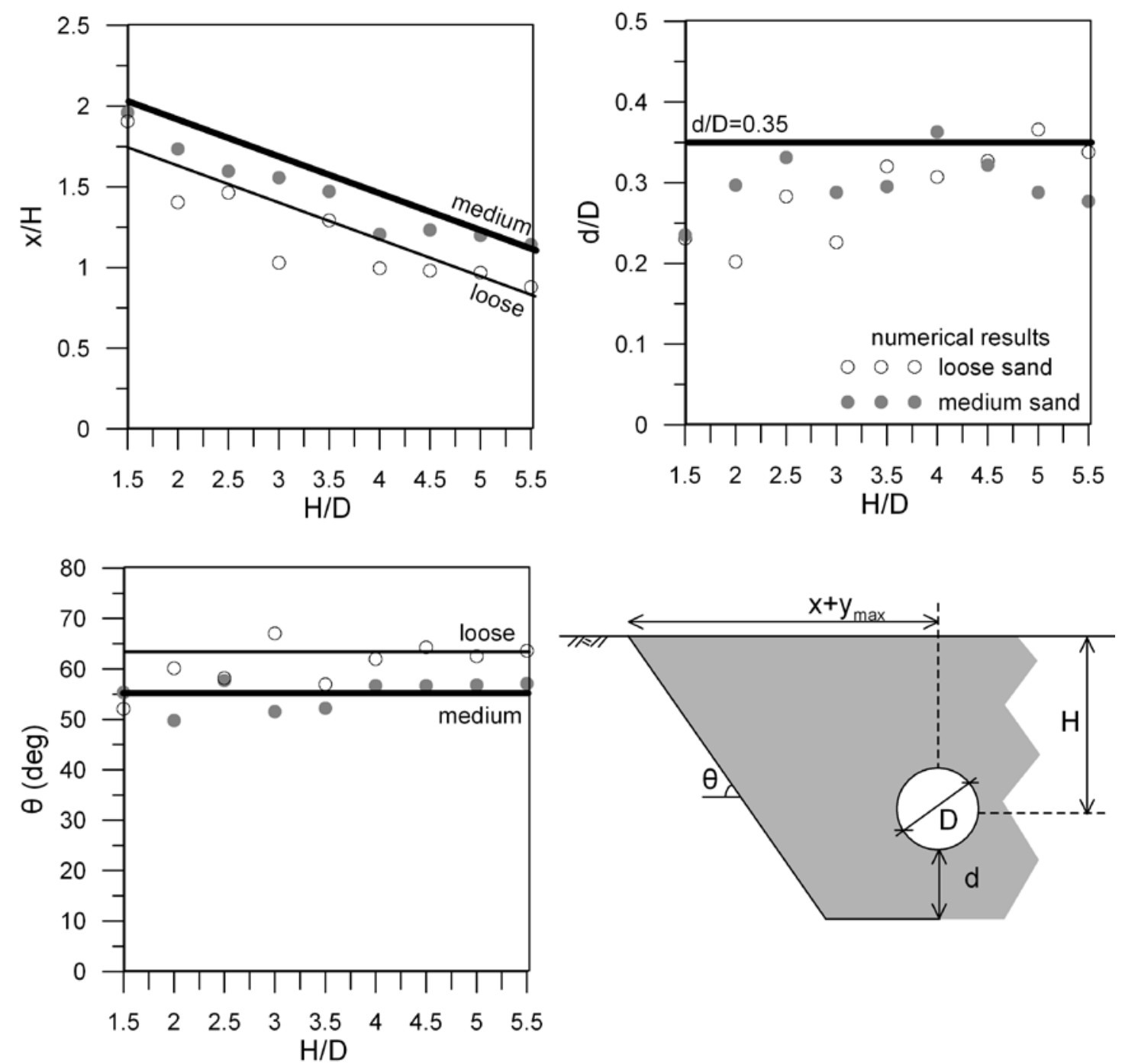

Fig. 6

\section{Comparison with other test results}

Karimian et al. [10] presented the results of full-scale experiments which were performed to investigate the use of geotextiles for reducing forces on pipelines from trench interaction effects. Of these experiments, we focus on the results from tests LN-1, LN-2 and LT-1. The first two tests are identical and involve pulling a $0.467 \mathrm{~m}$ diameter pipeline in uniform sand, while the third test is a pull-in test on the same 
pipeline constructed in a trench, formed by mounting an inclined steel plate in the test box (Fig. 7). The backfill material used in the tests was medium-to-dense Fraser River dry sand with a relative density of $D_{r}=75 \%$, which corresponds to a dry density of $\rho=1600 \mathrm{~kg} / \mathrm{m}^{3}\left(\gamma=15.7 \mathrm{kN} / \mathrm{m}^{3}\right)$. According to Karimian et al. [10], the peak friction angle of the material at $D_{r}=75 \%$ is $\varphi_{\text {peak }}=45^{\circ}$ and the critical-state friction angle is $\varphi_{\text {crit }}=33^{\circ}$. The maximum void ratio of the sand is $e_{\max }=0.94$, which yields a reference unit weight at $D_{r}=0 \%$ equal to $\gamma_{r e f}=13.6 \mathrm{kN} / \mathrm{m}^{3}$.

In all tests the embedment ratio was equal to $H / D=1.92$, within the range of application of the proposed methodology, while the maximum applied horizontal displacement was $\delta_{\max }=0.85 \mathrm{D}(400 \mathrm{~mm})$. The layout of the trench was similar to the one considered in this study, however, as illustrated in Fig. 7, its presence increased the maximum force on the pipeline by about $20 \%$ compared to the case of uniform backfill conditions. Karimian et al. reported that, as the imposed displacement increased, the pipeline moved along the inclined plate, translating not only horizontally but also vertically. Note that the vertical segments of the forcedisplacement curve measured during test LT-1 correspond to unloading and subsequent reloading of the pipeline, which according to Karimian et al. has no significant effect on the pulling force.

The numerical results are compared with the experimental measurements of Karimian et al. [10] in Fig. 7, considering the maximum predicted force on the pipeline and the trench dimensions. The methodologies proposed by Trautmann and O'Rourke [1] and the ASCE-ALA guidelines [2, 3] for $\varphi=45^{\circ}$ are also applied to estimate the maximum anticipated force. The upper limit of the peak force predicted by the Trautmann and O'Rourke nomograph [1] corresponds to a water-filled pipe, while the lower limit is for a gas-filled pipe. 
The proposed upper bound of the $N_{h}$ factor corresponding to medium sands (thick black line in Fig. 5) provides a reasonable estimate of the pipeline maximum force. Use of the approximate formula (Eq. 5) instead, while considering the density values $V$ and $\gamma_{\text {ref }}$ reported by Karimian et al. [10], would result in a 5\% higher force one the pipeline $(56.7 \mathrm{kN} / \mathrm{m}$ instead of $53.9 \mathrm{kN} / \mathrm{m})$. The nomograph proposed by Trautmann and O'Rourke [1] provides an upper bound to the forces developed, while the Hansen [2, 3] empirical method resulted in an unrealistically high estimate of about $160 \mathrm{kN} / \mathrm{m}$.
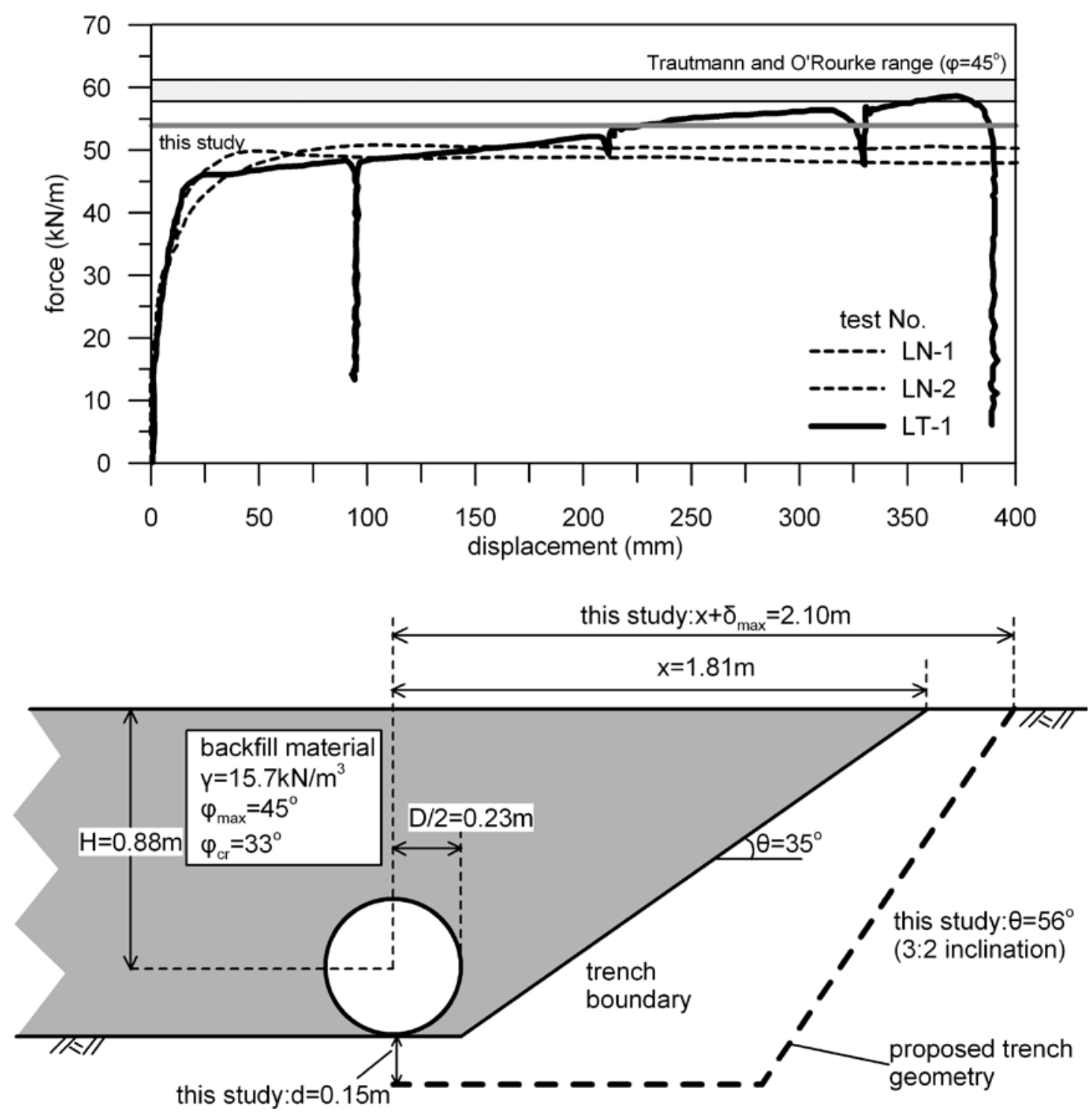

Fig. 7 
The trench dimensions required to eliminate interaction effects are estimated from the charts of Fig. 6 for a medium sand with $H / D=2$ (Fig. 7). The trench dimensions resulting from the proposed methodology are somewhat larger $\left(2.1 \mathrm{~m}^{2} /\right.$ running meter for the right half) than the one implemented in the tests $\left(1.3 \mathrm{~m}^{2} /\right.$ running meter for the right half), an indication that the increase in the pipe force recorded in test LT-1 could be mitigated by adopting an enlarged trench. Of course, more full-scale tests with different trench geometries, backfill material densities, and embedment ratios would contribute towards further substantiation of the proposed trench design guide.

\section{Concluding remarks}

The key contributions of the presented study can be summarized as follows:

- Comparison of new numerical results with published experimental measurements from full-scale tests on pipelines with a low embedment ratio $H / D$ suggest that the critical-state shear strength parameters of the dry sand are sufficient to describe the failure mode of the pipeline-backfill system; at least for loose-to-medium backfill, which will be the case for a pipeline embedded in a trench. A numerical formulation that can account for large backfill deformations via explicit integration and adaptive meshing provides a much better comparison with experimental results than previous published work, at least for the range of parameters considered.

- A direct implication of the above is that, for shallow pipelines buried in dry loose-to-medium sand, the backfill failure prism geometry and maximum forces developing on the pipeline are not strongly correlated to the peak friction and dilation angle of the sand. This can be used to derive an approximate formula for estimating the maximum horizontal force on the pipeline. The predictions from this formula complement those from the nomograph proposed by Trautmann and O'Rourke [1], for values of the 
embedment ratio $H / D$ which are of major practical interest. The proposed approach does not require laboratory measurement or empirical estimation of the peak friction angle of the backfill, merely its in-situ density. This is much easier to measure and control in situ.

- If a bilinear force-displacement curve is used in conjunction with elastoplastic horizontal springs for the numerical analysis of the pipeline under permanent ground displacements, a "yield" displacement of $0.2 D$ and $0.08 D$ for loose and medium sand, respectively could be considered as a reasonable, conservative approximation, based on the results presented in Fig. 4.

- Simple diagrams are proposed for dimensioning the trench in which the pipeline is to be installed, so as to ensure that failure will be restricted to the backfill. This avoids interaction with the possibly much stiffer surrounding natural ground.

- The predictions from the proposed design tools agree reasonably well with the test results of Karimian et al. [10]. The experimental and numerical results suggest that the geometry of the embedment trench must be determined carefully to mitigate interaction effects and minimise construction costs.

\section{References}

[1] Trautmann, C.H. and O' Rourke, T.D. Lateral force-displacement response of buried pipe. J. Geotech. Engng ASCE 1985;111, No. 9, 1068-1084.

[2] Hansen, J.B. The ultimate resistance of rigid piles against transversal forces. 1961; Bulletin 12, Danish Geotechnical Institute, Copenhagen, Denmark.

[3] American Lifelines Alliance ALA. Guidelines for the design of buried steel pipes. New York: ASCE; 2005.

[4] Olson, N. A. Soil performance for large scale soil-pipeline tests. PhD thesis, Cornell University, Ithaca, NY; 2009

[5] O' Rourke, T.D. Geohazards and large, geographically distributed systems. Géotechnique 2010; 60, No. 7, 505-543. 
[6] Yimsiri, S., Soga, K. Yoshizaki, K. Dasari, G. and O' Rourke, T.D. Lateral and upward soil-pipeline interactions in sand for deep embedment conditions. J. Geotech. Geoenviron. Engng ASCE 2004; 130, No. 8, 830-842.

[7] di Prisco, C. and Galli, A. Soil-pipe interaction under monotonic and cyclic loads: experimental and numerical modeling. Proceed. 1st Euro-Mediterranean Symposium on "Advances in Geomaterials and Structures", Hammamet, Tunisia; 2006.

[8] Turner, J.E. Lateral force-displacement behavior of pipes in partially saturated sand. MS thesis, Cornell University, Ithaca, NY; 2004.

[9] Paulin, M.J., Philips, R., Clark, J.I., Trigg, A. and Konuk, I. A full-scale investigation into pipeline/soil interaction. Proceed. $2^{\text {nd }}$ International Pipeline Conference, Calgary, Canada; 1998.

[10] Karimian, H., Wijewickreme, D. and Honegger, D. Full-scale laboratory testing to assess methods for reduction of soil loads on buried pipes subject to transverse ground movement. Proceed. $6^{\text {th }}$ International Pipeline Conference, Calgary, Canada; 2006.

[11] Abaqus/Standard - User's Manual - version 6.11. Dassault Systemes Simulia Corp.

[12] Bolton, M.D. The strength and dilatancy of sands. Géotechnique 1996; 36, No. 1, 65-78.

[13] Davis, E.H. Theories of plasticity and the failure of soil masses. In Soil mechanics: selected topics (ed. I. K. Lee), London: Butterworth; 1968, p. 341-380.

[14] Trautmann, C.H. and O' Rourke, T.D. Closure on: Lateral force-displacement response of buried pipe. J. Geotech. Engng ASCE 1987; 113, 940-943.

[15] Sadrekarimi, A. and Olson, S.M. Critical state friction angle of sands. Géotechnique 2011; 61, No. 9, 771-783.

[16] Jefferies, M.G. Nor-Sand: A simple critical state model for sand. Geotechnique 1993; 43, No. 1, 91-103. 